\title{
TEARS OF THE HUMERAL ROTATOR CUFF
}

\author{
A Radiological and Pathological Necropsy Survey
}

\author{
R. E Cotron, Nottingham, and D. F. Rideout, London, England
}

From the Bland-Sutton Institute of Pathology and Radiological Department, Middlesex Hospital, London

Pain and stiffness of the shoulder joint are extremely common symptoms which may follow trauma, often of minor degree. Quite often, however, the symptoms may have a somewhat insidious presentation after exertion or excessive use of the shoulder in various occupations and recreations. The clinical diagnosis commonly made is of a rotator cuff tear and the patients are often referred for a radiological examination to confirm the diagnosis and to exclude disease. The criteria for radiological diagnosis of a tear are, however, the subject of some dispute and many features ascribed to this condition are found in many persons with no history of recent injury and no relevant symptoms.

The clinical diagnosis of rotator cuff tear implies rupture of a number of the tendinous and fibrocartilaginous fibres which comprise the rotator cuff. This is a well defined anatomical structure into which the subscapularis, supraspinatus, infraspinatus, and teres minor

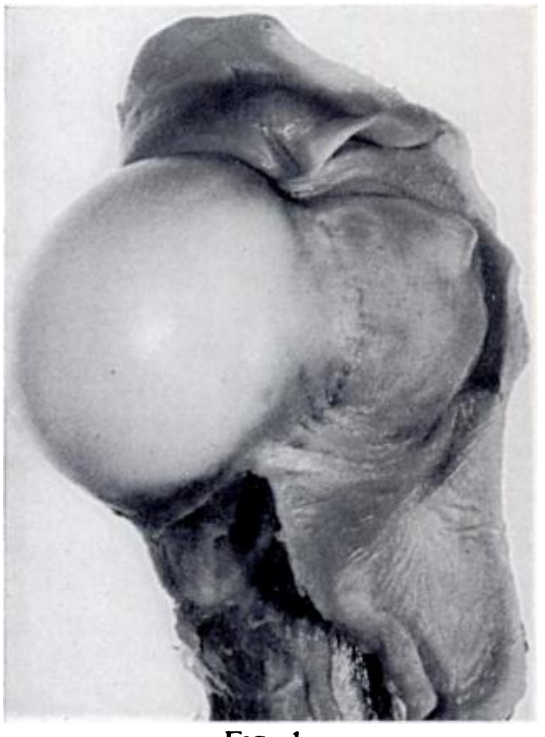

FIG. 1

Normal shoulder with the capsule reflected to show the inner aspect of the rotator cuff covered by smooth synovial membrane. muscles are inserted. The cuff itself is attached to the tuberosities of the head of the humerus along the upper two-thirds of the anatomical neck. The biceps tendon in its groove between the tuberosities passes under this attachment. The cuff therefore lies between the acromion and the greater tuberosity of the humerus and a non-communicating bursa is commonly found on the upper surface of the cuff at this point. On its inner aspect the fibres of the cuff are closely applied to the synovial membrane which is reflected at the insertion of the cuff to the margin of articular cartilage (Fig. 1).

In view of these anatomical relations it is not surprising that injury to the cuff, particularly by sudden and violent strain, may also cause damage to the biceps tendon, the acromion, the humerus at the tendinous insertion, the synovial membrane and the articular cartilage. Since symptoms are usually relieved by conservative measures and operation is seldom indicated, little information is available regarding the appcarances of the cuff and the joint in the acute stage, apart from radiological changes; also, few studies have been performed on patients at necropsy who had a clear history of this condition during life. In view of the natural history of the condition it is most likely that tears of varying severity may occur in many patients which are either unnoticed or forgotten as a past uncomfortable episode. Although this may seem surprising in view of the considerable derangement of structure found in the joints examined, it must be remembered that the shoulder is not a weight-bearing joint and gleno-humeral movement can be avoided by compensatory use of the scapula. This might account for radiological changes which are found in asymptomatic persons and yet be acceptable as evidence of previous injury.

Radiological diagnosis of rotator cuff tears has been made previously on the following findings. 1) Cystic appearances in the bone of the upper two-thirds of the anatomical neck 
of the humerus (Codman 1934, Harrison 1949, Golding 1962). 2) Recession, cortical irregularity, sclerosis or trabecular atrophy of the greater tuberosity of the humerus (Codman 1934, Harrison 1949, Golding 1962). 3) A distinct notch or gap between the articular surface of the head of the humerus and the greater tuberosity (Codman 1934, Harrison 1949, Golding 1962). 4) Narrowing of the acromio-humeral gap (Golding 1962). 5) Irregular new bone formation on the lateral margin of the acromion (Codman 1934).

Cysts in the anatomical neck and narrowing of the acromio-humeral gap have often been described as evidence of osteoarthritis of the shoulder joint but confirmatory evidence is lacking. Cysts of similar appearance also occur in the femoral neck and have been thought due to osteoarthritis and rheumatoid arthritis. It has been further suggested that osteophytes from degenerative change in the acromio-clavicular joint may cause rotator cuff tears by local pressure and friction (Watson-Jones 1952). Codman (1934) suggested that the rough edge of new bone along the lateral margin of the acromion may wear through the supraspinatus tendon and cause fraying and rupture of the fibres. He studied 200 shoulders from 102 necropsies in a geriatric hospital but without radiological examination. Most of these patients were between sixty and eighty years old, there being seventy-four men and twenty-eight women, and he found that the incidence of rotator cuff ruptures was 32 per cent. Codman also described avulsion, flattening, fraying and adhesion of the long head of biceps and "caverns" containing vascular tissue beneath the greater tuberosity. Sclerosis of bone at the cuff insertion, irregularity and recession of the greater tuberosity were also noted. In a further group of patients who had complained of shoulder pain Codman reported radiological and clinical findings, including the factors $1,2,3$ and 5 mentioned above, and he also commented that he had never seen a fresh rupture of the cuff at operation.

Harrison (1949) described the radiological and necropsy findings in the shoulder of a man of thirty-six. The radiological appearances of a cyst below the anatomical neck were correlated with a multilocular cavity lined by fibrous tissue which was subsequently found at a comparable site. This cavity communicated with the anatomical neck and there was an old tear of the subscapularis tendon. Harrison (1949) also investigated 745 patients with shoulder injury, 111 of whom had radiographs taken. He described factors 1, 2 and 3 as typical of rotator cuff lesions.

Golding (1962) described factors 1, 2, 3 and 4 as occurring in rotator cuff lesions in addition to sclerosis of the acromion. Cystic appearances were seen radiologically in some cases but at necropsy no obvious communication with the joint cavity via the anatomical neck was found. In these cases, however, the covering of bone was very thin and a pin could easily be pushed into the cavity through the cortical bone. The cystic lesions were subsequently demonstrated histologically.

It was considered that a combined radiological and pathological survey of shoulder joints at necropsy might clarify the significance and relative importance of the various factors accepted as radiologically diagnostic of rotator cuff tears and confirm that these changes were always associated with tears. Such a study might also correlate the severity of changes and provide information about their pathogenesis.

\section{MATERIAL AND METHODS}

Antero-posterior radiographs of both shoulders were taken in an unselected series of 106 necropsy subjects over a period of nine months at the Middlesex Hospital. In only two cases was there any record in the clinical notes of symptoms referable to the shoulders, but it should be stressed that no other attempt was made to elicit a history of such symptoms other than by reference to the notes. The axial view was not done because the rigidity of the cadavers made such a view impossible. Rigidity also caused slight variation in the position of the shoulder at the time of radiography. For the practical purpose of this study these variations are considered to be unimportant.

VOL. 46 B, NO. 2, MAY 1964 
In nearly all the cases, when any radiological abnormality was found the shoulder joint was removed from the body for dissection. This was done by reflecting the skin and dividing the soft tissue and bone at the middle of the clavicle, the neck of the scapula and the junction of the uppermost and middle thirds of the humeral shaft. After the joint capsule had been exposed by dissection the scapula was removed. The joint capsule was incised at its lowest attachment to the humeral neck and the capsule and rotator cuff reflected upwards over the tuberosities to expose the inside of the joint. After formalin fixation, specimens for histological examination were taken, and after freezing, the humeral heads were cut in serial. Any abnormalities were recorded and an attempt was made to grade the severity of the findings as slight, moderate or severe. In some cases further radiographs were taken of the specimens. When radiological abnormalities were seen in both shoulders the joint which appeared to be most affected was removed, but in a few instances both joints were explored. A small control series of six radiologically normal joints were also excised and examined.

In addition to the radiological findings already stated, note was made of radiographic evidence of calcification in the rotator cuff tendons, osteoarthritic changes in the acromioclavicular joints and sclerosis of the cortex of the inferior surface of the acromion.

\section{RESULTS}

Both shoulders were examined radiologically on 106 cadavers and abnormalities were found in sixty-eight shoulders-in both shoulders of thirty bodies and one shoulder of eight bodies. Thirty-six abnormal shoulders were removed from thirty-three bodies and all the specimens showed rotator cuff tears with the exception of one in which there was evidence of rheumatoid arthritis. The six shoulders which appeared to be radiologically normal were found to be anatomically normal. The ages of the bodies ranged from twenty-eight to ninety in those with abnormal findings, and from thirteen to eighty in those with normal findings. The incidence of male and female bodies was equal.

\section{RADIOLOGICAL FINDINGS}

The diagnostic criteria of cuff tears and the incidence of them in sixty-eight shoulders were: 1) Cysts in the upper two-thirds of the anatomical neck (fifty-six shoulders) (Fig. 2); 2) irregularity of the cortex of the greater tuberosity (thirty-one shoulders) (Fig. 3); 3) sclerosis of the greater tuberosity or of the groove between it and the articular surface (twenty-seven shoulders) (Fig. 4); 4) exaggeration of the groove between the greater tuberosity and the articular surface of the head of the humerus (eight shoulders) (Fig. 4); 5) narrowing of the acromio-humeral interval (eleven shoulders) (Fig. 5); 6) sclerosis of the humeral surface of the acromion process (nine shoulders) (Fig. 5).

Recession and trabecular atrophy of the greater tuberosity was more often found in the older bodies, many of which had otherwise normal films. Osteophytes on the inferior margin of the acromio-clavicular joints were found in only six cases and were not thought to be a factor in tears of the cuff. Three had otherwise normal radiographic appearances and three had evidence of tears subsequently confirmed by dissection.

Seven shoulders showed calcification in the rotator cuff tendons (Fig. 6), all of which were proved to have tears.

In one case of rheumatoid arthritis both shoulders showed loss of articular cartilage and erosion of the cortex, bilateral acromio-humeral narrowing and some lipping and sclerosis of the glenoid surface. Fibrous ankylosis with an articular pannus was subsequently discovered.

The acromio-humeral interval was measured from a thin line of dense cortical bone marking the inferior aspect of the acromion at a point directly above the head of the humerus and recorded as the smallest distance between this line and the articular cortex of the humeral 
head. Subjects with no radiological abnormality showed an acromio-humeral interval from 6 to 14 millimetres.

Seven necropsy specimens showed a full-thickness tear of the rotator cuff and of the bursa so that the head of the humerus articulated with the acromion. In these subjects the acromiohumeral interval was 1 millimetre in three cases, 2, 3 and 4 millimetres in three other cases and 9 millimetres in another case. The full-thickness tear was almost complete along its whole

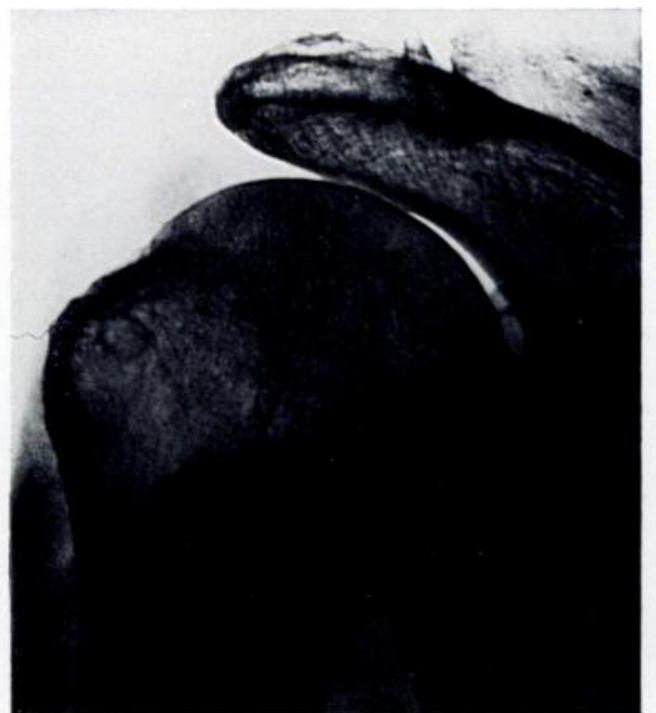

FIG. 2

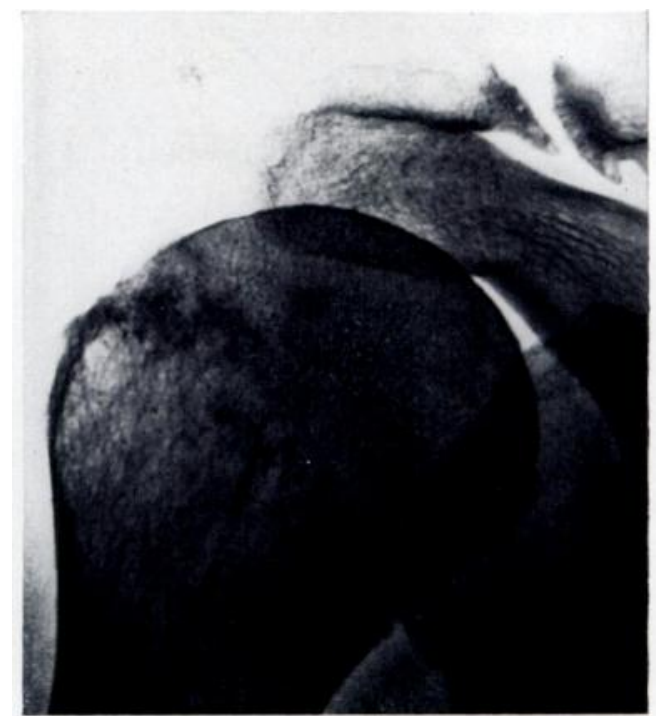

FIG. 3

Figure 2-A cyst in the greater tuberosity. The cortex of the greater tuberosity is sclerosed and very slightly irregular. The tear in this case was not severe. Figure 3-The cortex over the greater tuberosity is irregular and there are poorly defined cysts along the anatomical neck. The acromio-clavicular joint shows subarticular cyst formation and osteophytes at the inferior margins. The tear was severe.



FIG. 4

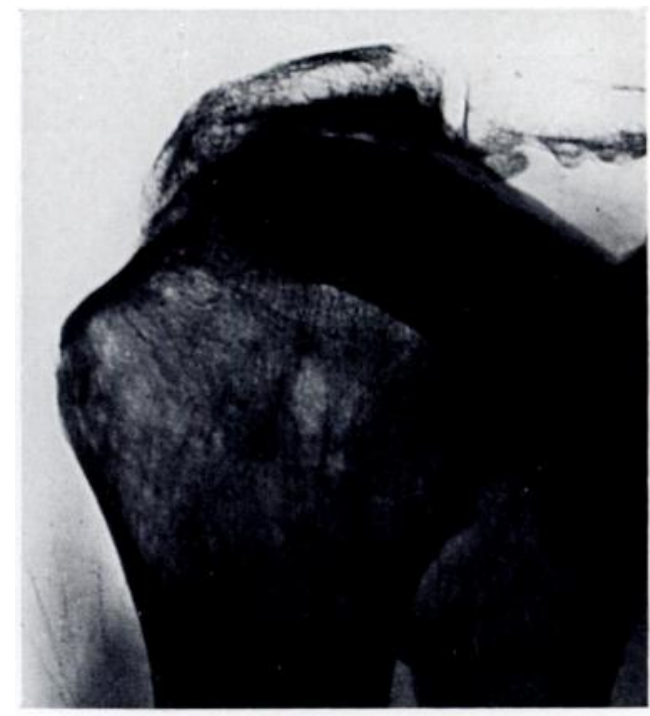

FIG. 5

Figure 4-The groove between the greater tuberosity and the articular surface is exaggerated with sclerosis of the underlying bone. The acromio-humeral gap is narrowed. Irregular cystic changes are present. Although the tear was severe acromio-humeral articulation was not present. Figure 5-Cysts are present and the cortex of the greater tuberosity shows sclerosis. The acromio-humeral gap is narrowed and there is sclerosis of the inferior surface of the acromion. Acromio-humeral articulation was present. 
attachment in four cases in which the interval was between 1 and 4 millimetres, incomplete behind in a case with an interval of 2 millimetres, and incomplete in front in two cases with intervals of 3 and 9 millimetres.

In other radiologically abnormal shoulders the acromio-humeral interval was between 2 and 11 millimetres. The case with rheumatoid arthritis had intervals of 4 and 5 millimetres.

Of the seven cases with acromio-humeral articulation, six showed radiological sclerosis of the inferior surface of the acromion. Only three other shoulders showed this change and these were the opposite shoulders of those cases with acromio-humeral articulation. Irregularity of the bone at the lateral margin of the acromion was found in three cases, in all of which

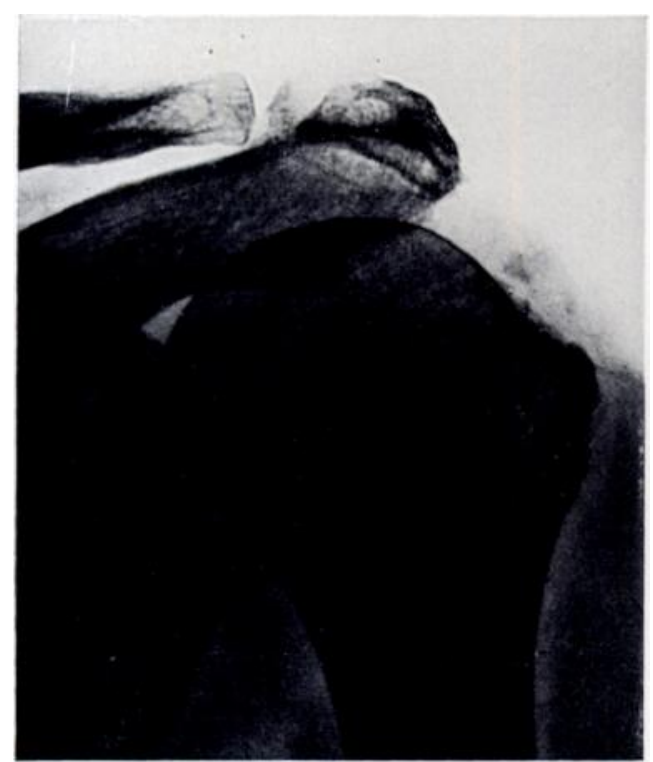

FIG. 6

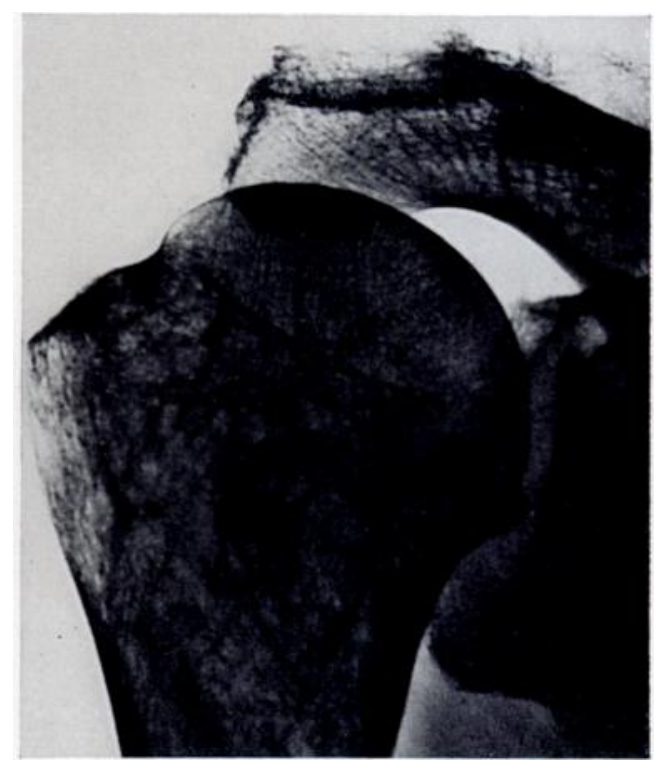

Fig. 7

Figure 6-Calcification in the rotator cuff and irregularity of the greater tuberosity. A cyst can be seen under the articular surface. A severe rotator cuff tear was present. Figure 7-Irregularity of the bone at the outer end of the acromion and to a lesser degree over the greater tuberosity. The cortex over the greater tuberosity and the groove dividing it from the articular surface is sclerosed. There are irregular cystic appearances along the anatomical neck. The rotator cuff tear was complete but acromio-humeral articulation was not present.

complete tears of the cuff were demonstrated (Fig. 7). This feature was not seen in other shoulders.

In two cases an unusually thick and apparently sclerotic cortex of the greater tuberosity, which was bilateral but not symmetrical, was the only radiological abnormality (Fig. 8). The sclerosed area had a smooth and regular surface. Both shoulders were removed and found to be entirely normal and these have been included in the figures of the radiologically normal.

Diagnostic radiographic changes of osteoarthritis were found in two shoulders with bony lipping of the inferior articular margin of the humeral head in both and some eburnation of the glenoid in one (Fig. 9). Both subsequently showed rotator cuff tears. Pathological examination disclosed osteoarthritic changes in fifteen out of thirty-three cases, severe in four, moderate in two, and mild or early in nine. Nearly all these patients were seventy years of age or more and the presence or absence of osteoarthritic changes did not correlate to any significant extent with the presence or absence of rotator cuff tears.

The shoulder of the patient with the previous fracture showed impaction deformity and new bone formation at the fracture margins. Small subcortical cysts were also found in the region of the anatomical neck and a partial tear of the rotator cuff was subsequently found. 
Of the subjects showing radiological cysts in the anatomical neck of the humerus and subsequently pathologically examined, all were found to have rotator cuff tears. The cysts were always in a similar situation but varied considerably in numbers and in size (Figs. 2 and 7). Most were solitary and immediately subcortical, although when multiple (up to three in a few cases) some were occasionally found a little distance from the cortex of the upper humeral head. This site of cyst formation is immediately beneath the insertion of the inner fibres of the rotator cuff and, in all cases examined, readily recognisable tears were found in the tendinous fibres, particularly those of the inner aspect of the cuff. Cysts were not found in the absence of tears.

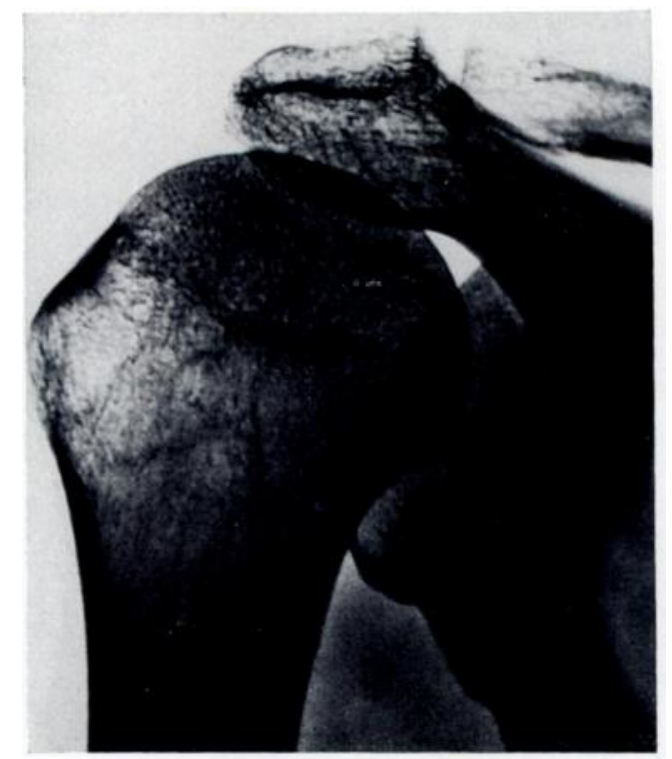

Fig. 8

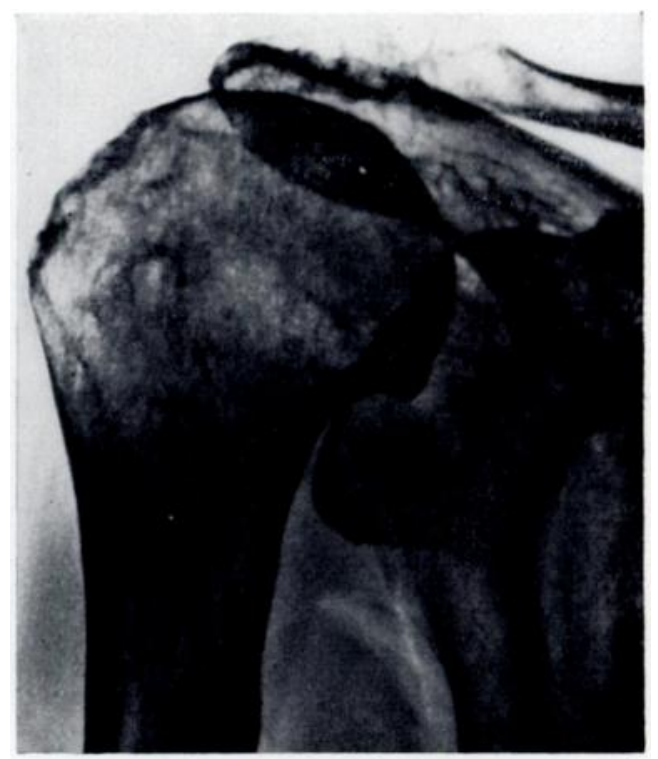

Fig. 9

Figure 8-The cortex of the greater tuberosity is sclerosed but no other changes can be seen and no abnormality was found in the shoulder at necropsy. Note the thin line of the normal inferior acromial cortex. Figure 9-Acromio-humeral narrowing with sclerosis of the inferior surface of the acromion. The cortex of the greater tuberosity is irregular and there are cysts along the anatomical neck. The inferior articular margin of the humeral head is lipped. There was a complete tear with acromio-humeral articulation.

In seventeen shoulders with severe tears four showed sclerosis and irregularity of the greater tuberosity, three had sclerosis only and there was irregularity only in seven; in three these features were not radiologically demonstrated. In sixteen cases with less severe tears two showed irregularity and sclerosis, one irregularity alone and five sclerosis alone: eight did not show either feature.

\section{PATHOLOGICAL FINDINGS}

Normal shoulders-All six radiologically normal cases showed no structural abnormality. The rotator cuffs had a smooth inner surface lined by the synovial membrane of the joint and there was a smooth transition from articular surface on to the reflected capsular tissues. Occasionally, slight folds were found in the synovial membrane.

Rotator cuff tears-These were found in all cases in which the radiological criteria suggested their presence. They varied considerably in their severity, being graded as incomplete of slight, moderate or severe degree or, in eight cases, complete and full thickness. The slight tears showed as irregularity of the inner surface with fraying of the fibres usually just in front of or behind the biceps tendon (Fig. 10). In moderately severe tears the inner fibres, usually on both sides of the biceps tendon, were more definitely ruptured with a small gap (Fig. 11), and in severe incomplete tears all but the outer fibres were ruptured usually over a considerable 


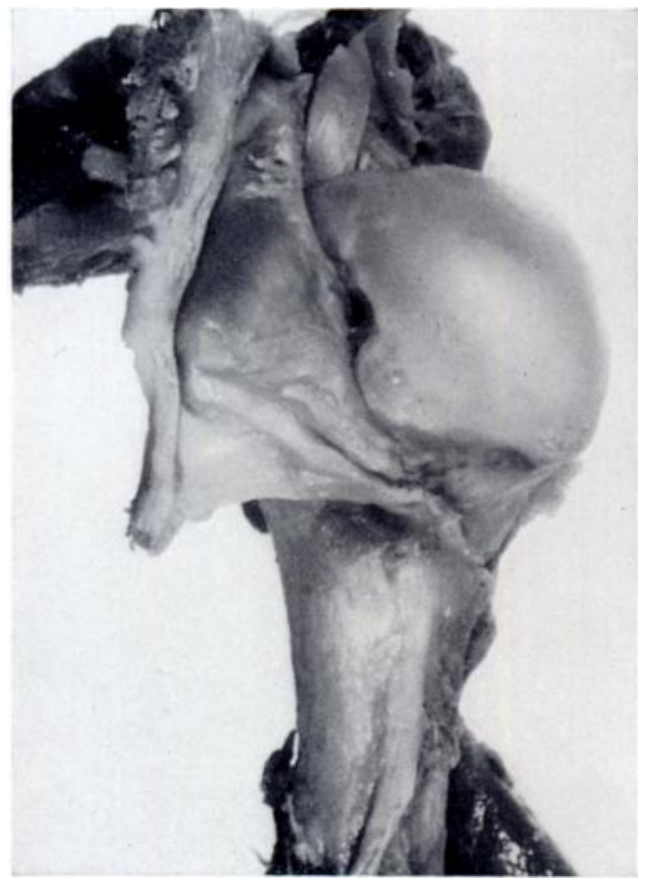

Fig. 10

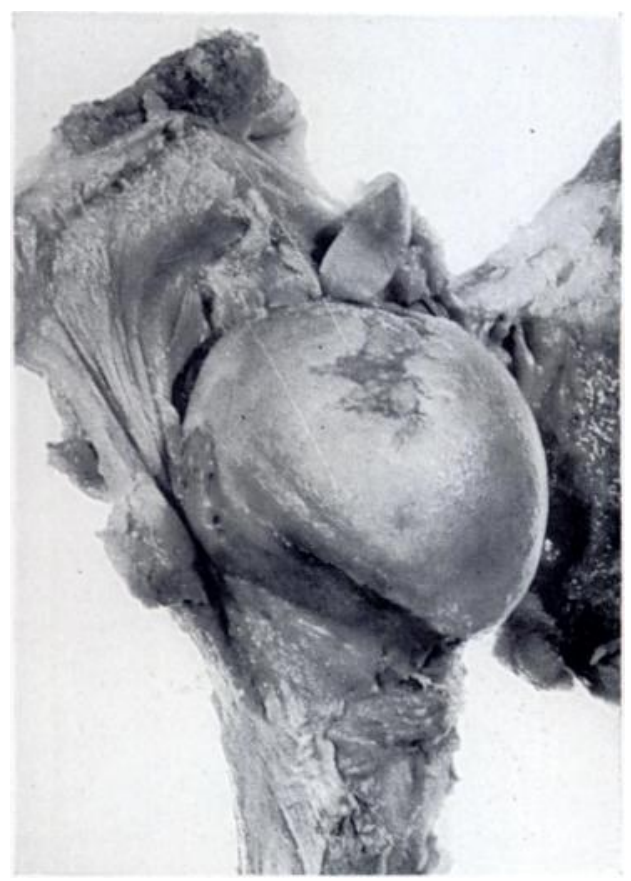

FIG. 11

Figure 10-A slight rotator cuff tear with fraying of the inner fibres near the biceps tendon. Avulsion of the fibres has resulted in a groove at the articular margin with a central " pore." There was a cyst present in the underlying bone. Figure 11-A moderately severe tear of the cuff with avulsion of the inner fibres. Small " pores" are visible in the anatomical neck and the joint shows osteoarthritic change.

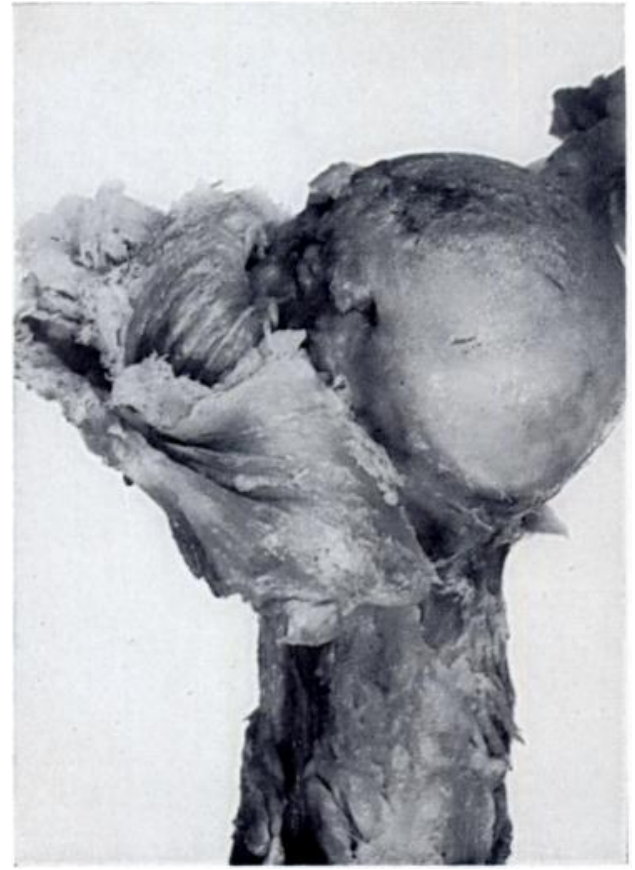

FIG. 12

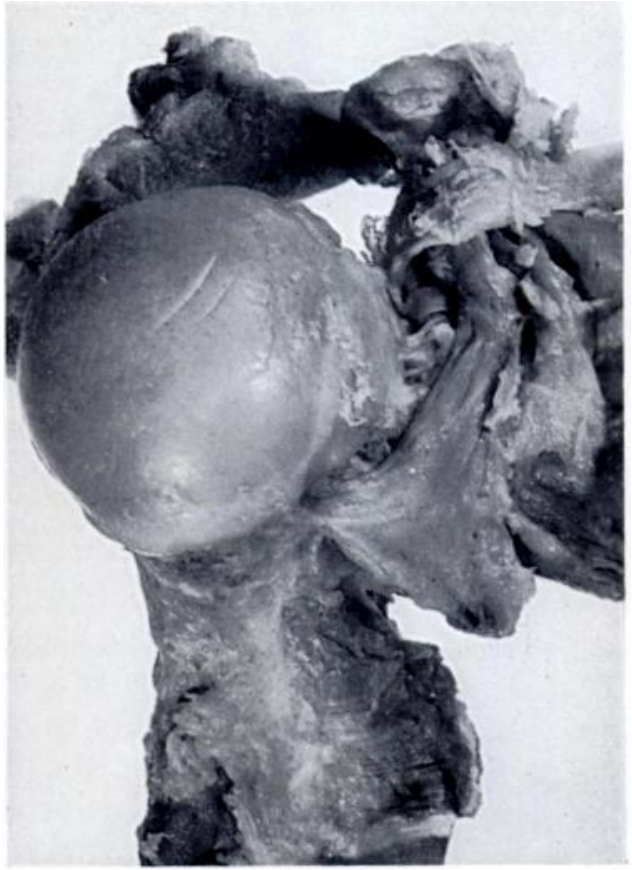

Fig. 13

Figure 12-A severe incomplete tear with marked irregularity of bone of the anatomical neck associated with avulsion of the fibres. The head of the humerus shows osteoarthritic change. Figure 13-Complete full-thickness tear of the rotator cuff. In areas of residual attachment anteriorly there are " bridges" of tendinous tissue and the biceps tendon shows marked fraying. 
length of the cuff (Fig. 12). Complete tears involved the full thickness of the cuff and the subacromial bursal wall (Fig. 13) leaving a large gap which allowed articulation between the head of the humerus and the undersurface of the acromion (Fig. 14). This articulation was seen in seven of the eight cases of complete tear.

In most cases the tear was greatest close to the insertion of the tendinous fibres into the humeral head. Foci of calcification in the region of tears were seen radiologically in seven cases and their presence confirmed histologically (Fig. 15). The ruptured fibres and those adjacent to the tear nearly all showed loss of structure with hyalinisation and areas of myxomatous degeneration (Fig. 16), but these changes were only found in the region of tears and were not found in their absence. It appears unlikely that such changes are primary ones but are secondary to the presence of the tear.

Other abnormalities were found almost constantly to be associated with tears. Rupture and detachment of the inner fibres of the cuff were uneven leaving narrow bands or " bridges" of tendinous tissue at the original site of attachment (Fig. 17). These increased in number with the severity of the tear but were not present in complete tears. In the slight tears they occasionally represented the only site of rupture of fibres.

A further associated abnormality was irregularity of the cortical bone and the margin of the articular surface at the site of insertion of the tendinous fibres, particularly when the tears were severe and considerable areas of the cuff had been avulsed. In this way a groove, sometimes deep, was apparent with undermining of the articular margin and increase in the distance between the articular margin and the greater tuberosity (Fig. 18). The margin of the articular cartilage was sharp and the bone in the floor of the groove commonly pitted and irregular. The appearances suggested either avulsion or resorption of a flake of bone. Bony loose bodies were not seen in any of the joints examined. The degree of this avulsion corresponded accurately

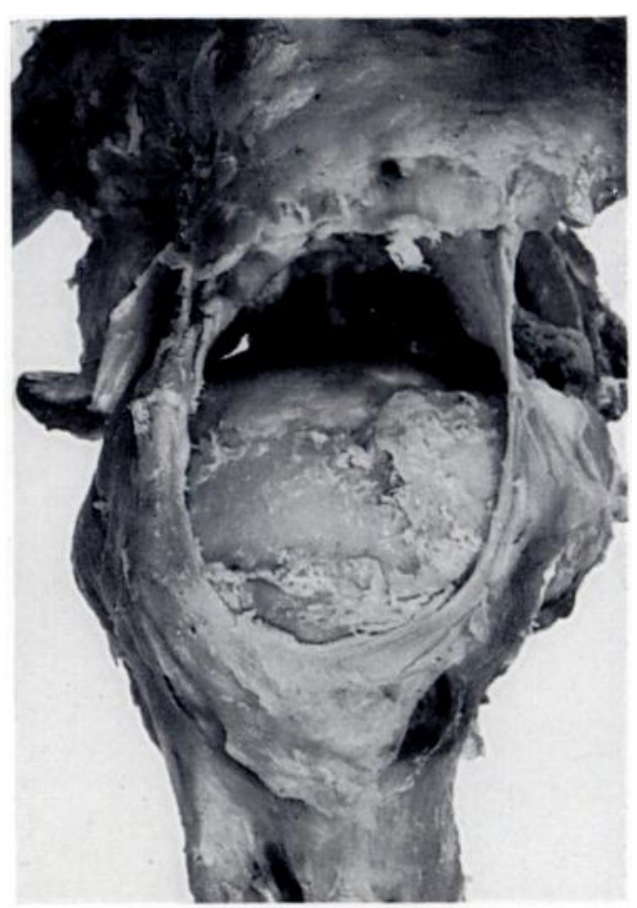

FIG. 14

Shoulder viewed from the lateral aspect showing a complete tear of the cuff with erosion of the articular surface of the humerus. Acromiohumeral articulation was present.

with the severity of the tear in the rotator cuff fibres. These features gave rise to a characteristic radiological appearance of sclerosis of the bone in the groove between the greater tuberosity and the articular surface in twenty-seven shoulders and exaggeration of the grooves in eight.

Cysts-Of the thirty-six abnormal shoulders removed, thirty-five showed radiological evidence of cysts in the upper humeral head and their presence was subsequently confirmed by dissection. All had rotator cuff tears of variable severity. The only abnormal shoulder which did not have cysts was from the case of severe rheumatoid arthritis.

The position of the cysts was constant being in the subcortical bone of the anatomical neck of the humerus immediately beneath the site of insertion of the inner fibres of the rotator cuff (Fig. 19). They varied in number from one to three but were usually solitary. When multiple some were one centimetre or more from the cortical margin. Their size varied from two millimetres to one centimetre across. Neither the number nor the size of the cysts bore a constant relationship to the severity of the tears. Cysts were present in joints which showed no evidence of osteoarthritis. 


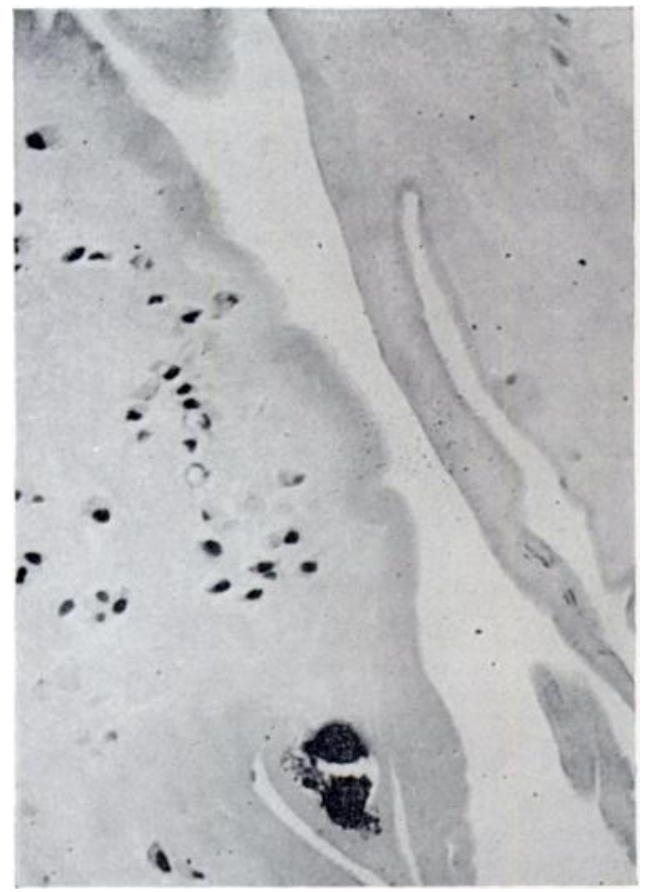

FiG. 15

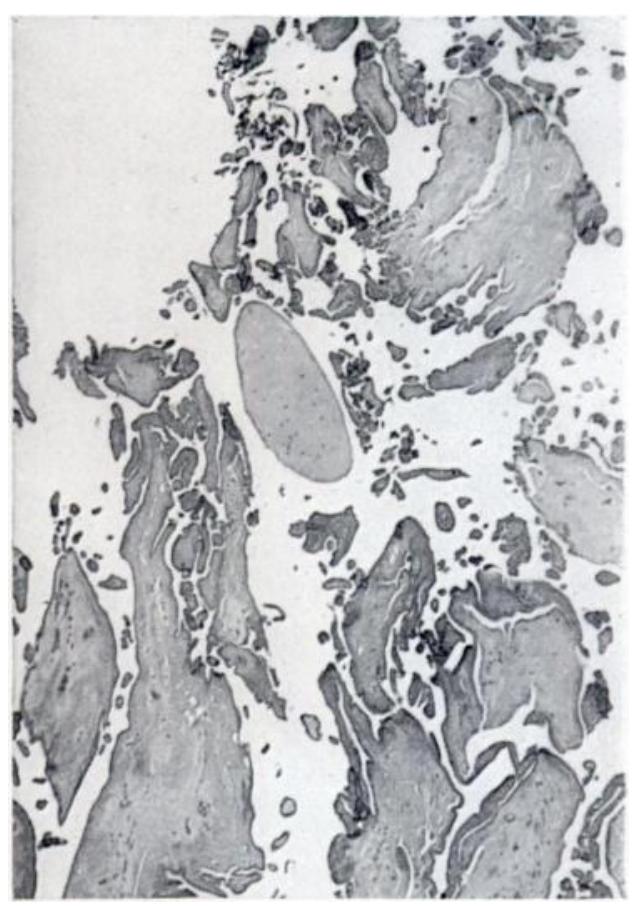

FIG. 16

Figure 15-Section showing a focus of calcification in torn rotator cuff fibres which also show myxomatous degenerative changes. (Haematoxylin and eosin, $\times 200$.) Figure 16-Section showing gross disorganisation of torn cuff fibres and areas of degeneration at the site of a tear. (Haematoxylin and eosin, $\times 30$.)



FIG. 17

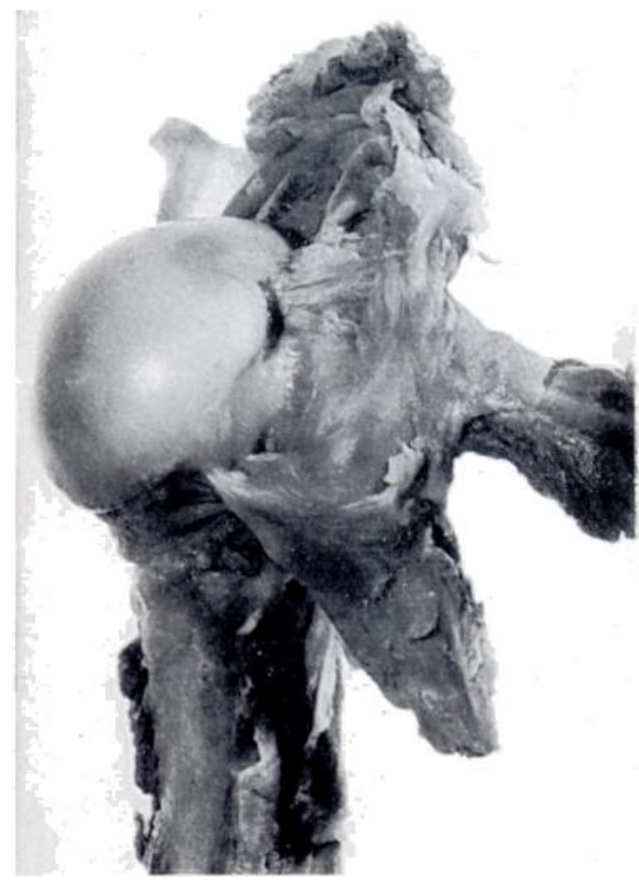

Fig. 18

Figure 17-The shoulder shows a moderately severe tear with multiple " bridges" of tendinous tissue. Figure 18-A deep groove at the articular margin at the site of a partial tear. 




FIG. 19



FIG. 20

Figure 19-Longitudinal section through the head of the humerus showing a bilocular cystic cavity in the subcortical bone with a "pore." Figure 20-A " pore " and a subcortical cyst. The inner aspect of the attached rotator cuff shows marked irregularity.

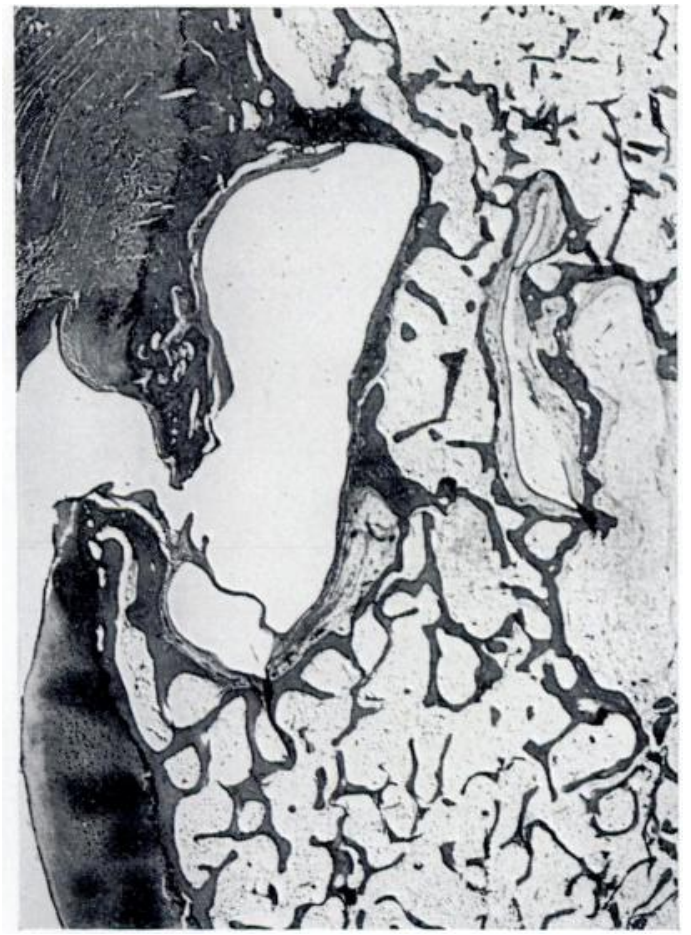

Fig. 21

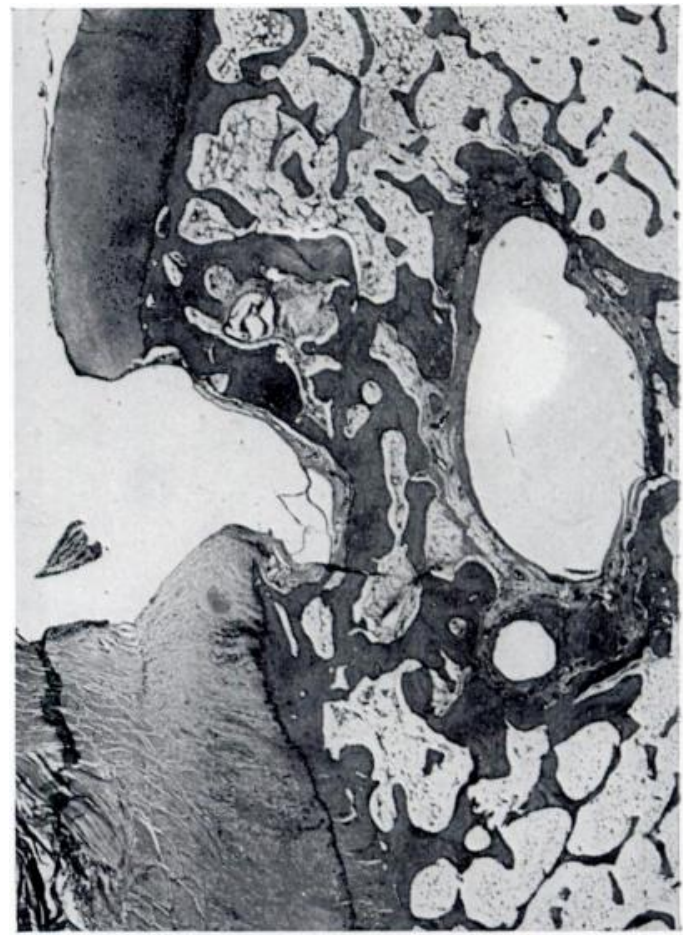

Fig. 22

Figure 21-Section through a "pore" which demonstrates continuity with the cystic space. This particular specimen showed a continuous lining of synovium. There is a second, deeper, cystic lesion. (Haematoxylin and eosin, $\times 6 \cdot 5$.) Figure $22-$ Sclerotic bone surrounds the cyst. The cortical bone is distorted and largely missing. (Haematoxylin and eosin, $\times 8$.) 


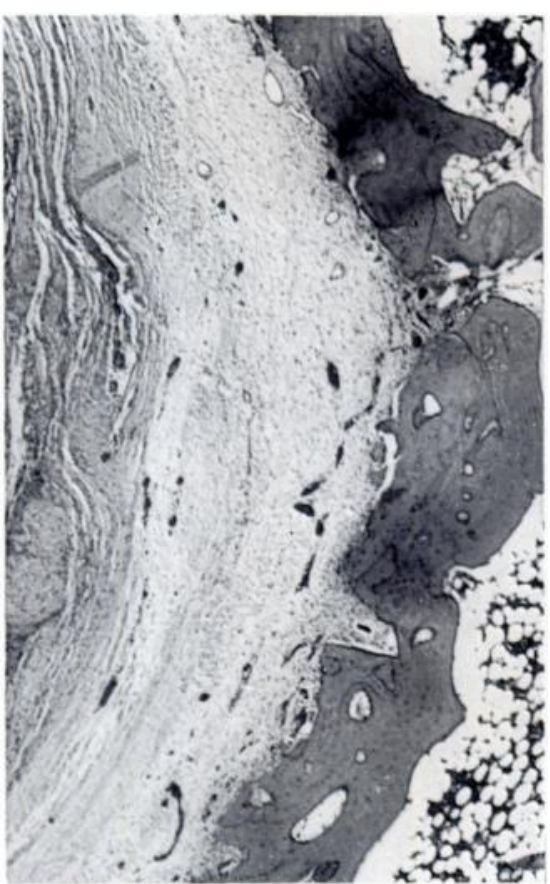

Fig. 23

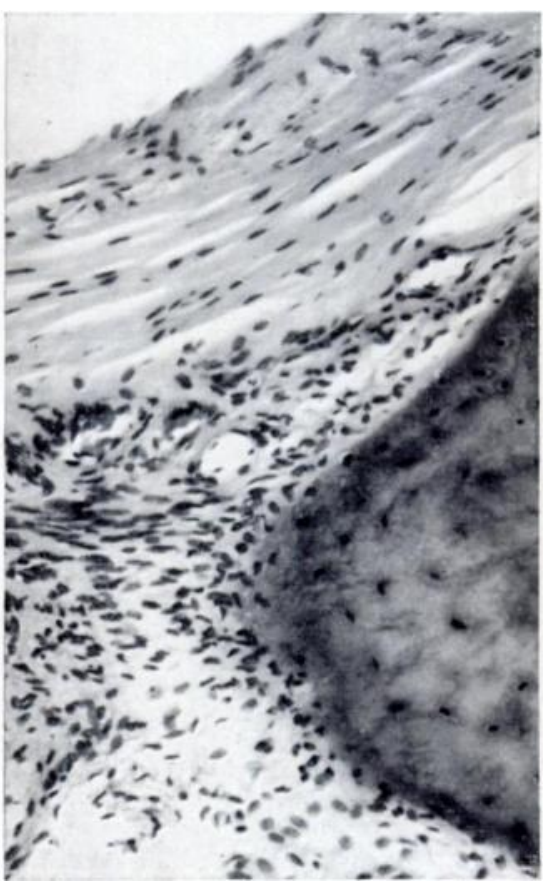

FIG. 24

Figure 23-The cyst wall is composed of connective tissue of moderate vascularity. The bony margin shows considerable new bone formation with numerous cement lines. (Haematoxylin and eosin, $\times 30$.) Figure $24-$ Cyst lining showing strands of collagen and fibroblastic and osteoblastic activity. (Haematoxylin and eosin, $\times 160$.)

In all but two subjects the cysts communicated with the shoulder joint by gaps in the bony roof which appeared either as "pits" (Fig. 20) or definite "pores" when viewed from the joint surface. These communications varied from pin-point in size (Figs. 21 and 22) to a maximum of three millimetres in diameter. In the two instances where no communication

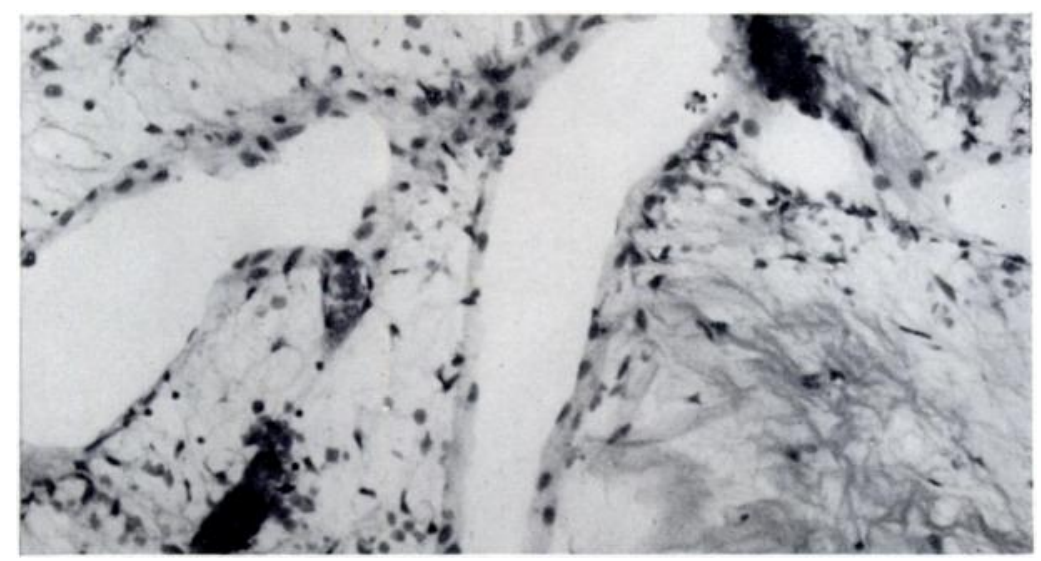

Fig. 25

Showing loose connective tissue with spaces lined by somewhat flattened cells of mesothelial appearance. (Haematoxylin and eosin, $\times 160$.)

was found the bony roof was extremely thin so that a pin could readily be pushed through into the underlying cavity in the manner described by Golding (1962). The pores were always situated in the anatomical neck of the humerus. 


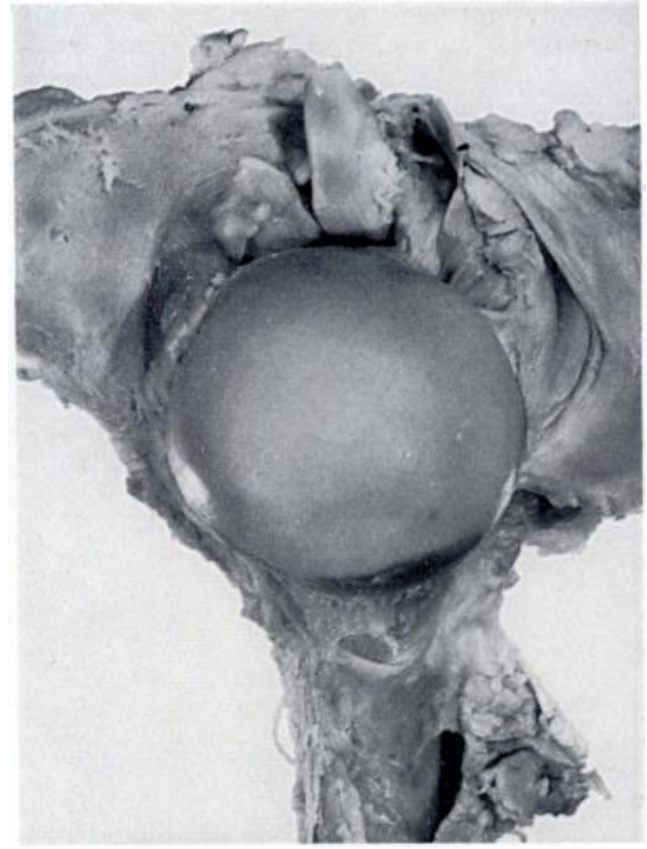

FIG. 26

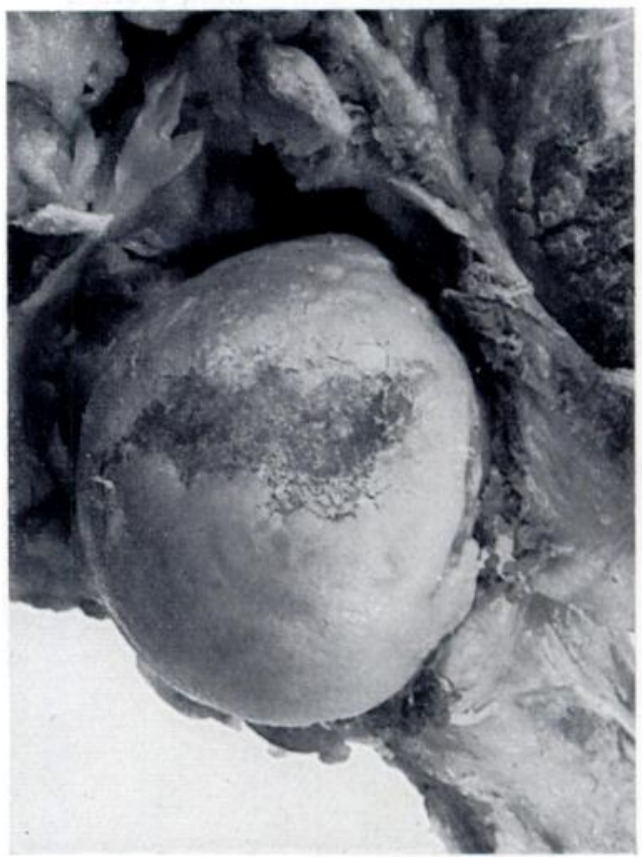

Fig. 27

Figure 26-The biceps tendon shows fraying of one border at the site of a rotator cuff tear. Figure 27Osteoarthritis of the head of the humerus and villous proliferation of the synovial membrane.

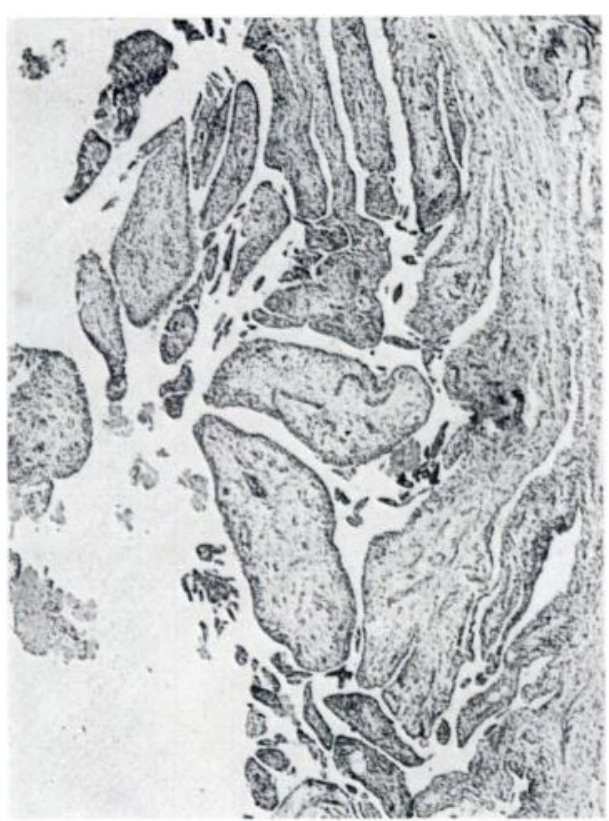

FIG. 28

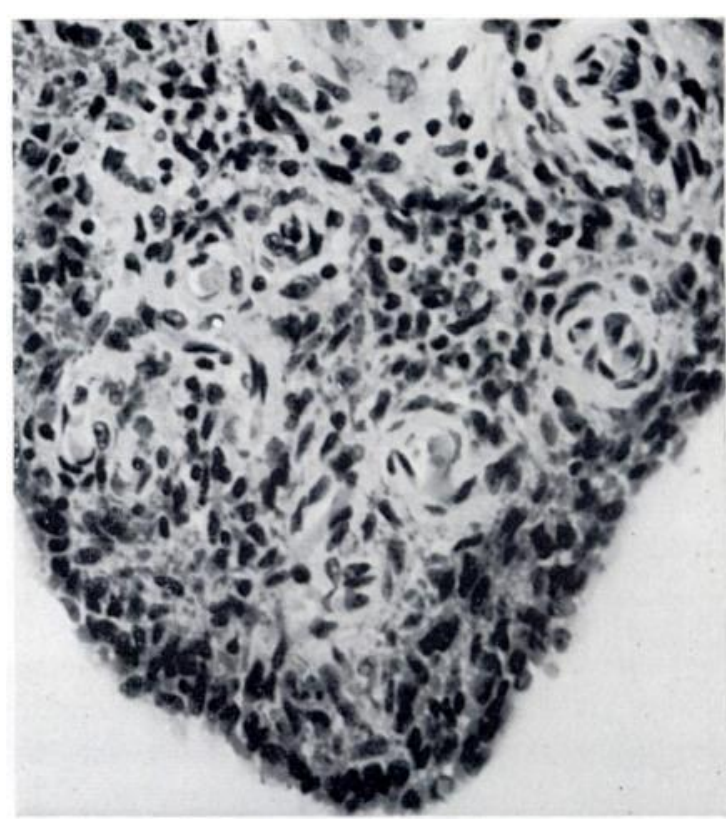

Fig. 29

Figure 28-Marked villous proliferation of the synovial membrane. (Haematoxylin and eosin, $\times 30$.) Figure 29-Pigmented villous proliferation with a cellular villus containing abundant iron-containing pigment granules. (Gomori's method for haemosiderin, 240.) 
The term cyst was perhaps not always correctly applicable to these lesions for while some consisted of a definite cavity lined by vascular connective tissue (Figs. 23 and 24), others showed a bony defect filled with fibrous tissue and fibrinous material in which there were several small spaces (Fig. 25). The surrounding bone varied from markedly sclerotic to grossly porotic but in many cases was of more normal structure. The tissue forming the roof of the defect was mostly of rather thin cortical bone with gaps at the sites of the "pores" but in a few instances it was composed of fibrous tissue only. The cystic spaces were lined by a layer of rather flattened cells, possibly mesothelial, and in a few cases a definite synovial lining was evident in continuity with that of the shoulder joint.

Biceps tendon-The biceps tendon showed pathological abnormality in eleven specimens, in each case associated with tears irrespective of the presence of osteoarthritis. Lesions varied from fraying, flattening and narrowing of the tendon (Fig. 26) to complete rupture in four cases, three of which had complete cuff tears with acromio-humeral articulation; the fourth case had a very severe, almost complete, tear.



Fig. 30

Showing profuse haemosiderin granules in phagocytic cells. (Gomori's method for haemosiderin, $\times 600$.)

Synovial membrane-In addition to distortion and destruction of the synovial membrane at the site of tears, fourteen cases showed varying degrees of villous synovial proliferation, mostly marked (Fig. 27). All these were associated with severe rotator cuff tears. Although in ten cases this was associated with osteoarthritis in the joint, there were five cases of osteoarthritis which did not show gross synovial abnormalities and four cases of synovial proliferation in the absence of osteoarthritic change.

In nine specimens the abnormality was a simple non-specific reactive villous overgrowth with an infiltration of chronic inflammatory cells, presumably related to the damage which caused the tears (Fig. 28). Five specimens showed very marked brown pigmentation of the synovium at the site of villous proliferation with, in the case of the two more marked examples, fusion of villi to form nodules. The pigment was found to be iron and the histological appearances were similar to those of pigmented villonodular synovitis (Figs. 29 and 30). In none of the cases did the changes approach the obvious tumour-like proportions of this condition as seen typically in the knee joint.

\section{DISCUSSION}

The radiological and pathological features described as associated with rotator cuff tears are common in necropsy specimens. In this series of 106 bodies they were present in thirty-eight and confirmed pathologically in thirty-five cases, usually being in both shoulders. The average 
age of those with abnormal shoulder joints was $70 \cdot 7$ years and of the radiologically normal $56 \cdot 25$ years. Although slightly more common in men there was no significant difference in sex distribution compared with the normals. The older age of patients with rotator cuff tears might be due to several factors. If trauma is solely responsible for the lesion it might be expected that the ligamentous tissues involved would be more susceptible with advancing age and with corresponding reduction in the speed and efficiency of the reparative processes. Alternatively it may be that the majority of these patients acquire their lesions from repetitive minor episodes of trauma over many years but with increasing deterioration on each occasion. This would certainly fit with the lack of clinical evidence of acute injury in many patients. What is more difficult to assess is the part played, if any, by degenerative disease affecting the rotator cuff and adjacent structures. Certainly it has been shown that there is no relationship to osteoarthritic change in the joints and although the tendinous fibres show some myxomatous degeneration next to the torn fibres, such a change may be secondary to the tear rather than primary.

Rotator cuff tears are constantly related with cyst formation in the anatomical neck of the humerus, partial avulsion of the tendinous insertion of the cuff, loss of the marginal articular surface, or excessive grooving at this margin with " bridge formation " due to uneven detachment of fibres on the inner aspect. Damage to the biceps tendon is also commonly found but osteoarthritic changes are not related, and it is of interest that the case of rheumatoid arthritis did not show any cysts in the humeral head. Severe tears with complete rupture of the cuff lead to additional articulation between the upper surface of the humeral head and the undersurface of the acromion. The cysts are situated in the subcortical bone immediately next to the site of detachment of the cuff fibres and in most cases the cyst roof is deficient to form " pores" communicating with the joint space.

It seems likely that all these changes are explicable on a basis of previous trauma but whether this results from a solitary severe incident or many minor episodes is a matter for conjecture.

Sclerosis of the greater tuberosity in the absence of any other radiographic evidence of a rotator cuff tear is an unreliable sign. In the two cases examined in this series no abnormality was found at necropsy.

Golding (1962) measured the interval between the acromion and humerus in 150 cases and gave the normal range as seven to thirteen millimetres. In this series the range was six to fourteen millimetres in shoulders that were radiologically normal. The narrowing of this interval is not an entirely reliable sign of a full-thickness tear of the cuff and wall of the bursa. One shoulder with a full-thickness tear showed an interval within the normal range. It was narrowed in the case of rheumatoid arthritis and in two cases with severe but incomplete tears. Sclerosis of the inferior acromial surface was a more reliable sign of acromial articulation.

Apart from the abnormalities associated with complete rupture of the cuff or acromial articulation, the radiological changes give little indication of the severity of the tear. Nevertheless, irregularity of the cortex of the greater tuberosity was three times more common in severe tears ( 65 per cent) than in less severe tears ( 20 per cent).

Irregular new bone formation was seen at the lateral acromial margin in only three shoulders, all of which had full-thickness tears. With this low incidence it is unlikely that this feature plays a significant part in the etiology of tears.

The exact mechanism of the production of the subcortical humeral cysts is unknown. Their close relationship in situation with tears of the cuff and avulsion of inner cuff fibres is certainly suggestive of a traumatic etiology. The stress might be transmitted through the cortex to produce direct disruption of subcortical cancellous bone. In such an event one would expect to find evidence of callus formation and this was not found. A further explanation could be based on inflammatory reaction to the presence of synovial fluid in the subcortical

vol. 46 B, NO. 2, MAY 1964 
bone which gains entry through the "pores" found after avulsion of rotator cuff fibres. However, no pores were found in two cases, although this does not exclude undue porosity of the bone which cannot be demonstrated macroscopically.

While non-specific reactive villous hyperplasia of the synovial membrane is not unexpected in damaged joints, the finding of pigmented villous hyperplasia is of some interest. Although the changes were not as florid as the tumour-like process seen in other joints, they may represent an intermediate stage of development of this lesion in a non-weight-bearing joint. This would lend some support to those who regard pigmented villonodular synovitis as a reactive rather than a neoplastic process and suggest that trauma and haemorrhage may be the etiological agents.

\section{SUMMARY}

1. Radiographs of both shoulders were performed on 106 unselected necropsy subjects and those found to be abnormal were examined pathologically.

2. Radiological abnormalities were found in sixty-eight shoulders of thirty-eight subjects. Pathological examination showed rotator cuff tears and associated abnormalities in thirty-five of these, rheumatoid arthritis in one, a previous fracture in one, and one was not examined. 3. The criteria for radiological diagnosis of rotator cuff tears are examined and discussed.

4. The radiological changes give little indication of the severity of the tears or associated abnormalities except in the case of complete rupture of the cuff when acromio-humeral articulation occurs.

5. The lesions are all explicable on a traumatic basis. There is no correlation with the presence or absence of osteoarthritic disease of the joint.

6. The biceps tendon may become damaged or even ruptured in this condition.

7. Villous synovial proliferation was found in fourteen cases, in five of which it was pigmented with histological appearances resembling pigmented villonodular synovitis. The significance of this finding is briefly discussed.

We would like to thank Dr F. Campbell Golding for his interest and helpful criticism in performing this survey and the post-mortem room and histological technical staff of the Bland-Sutton Institute of Pathology for their indispensable assistance and cooperation. Our thanks are also due to $\mathrm{Mr} \mathbf{M}$. Turney and the staff of the Middlesex Hospital Photographic Department for help with the illustrations.

\section{REFERENCES}

Codman, E. A. (1934): The Shoulder. Boston, Massachusetts: Thomas Todd Company.

Golding, F. C. (1962): The Shoulder-the Forgotten Joint. British Journal of Radiology, N.S. $35,149$.

Harrison, S. H. (1949): The Painful Shoulder. Journal of Bone and Joint Surgery, 31-B, 418.

WATSON-Jones, Sir R. (1952): Fractures and Joint Injuries. Fourth edition, pp. 445-446. Edinburgh and London:

E. \& S. Livingstone Ltd. 